\title{
Prospective assessment of cardiovascular risk parameters in patients with rheumatoid arthritis
}

\author{
Bożena Targońska-Stępniak ${ }^{1 *}$ (D, Mariusz Piotrowski ${ }^{1}$, Robert Zwolak', Anna Drelich-Zbroja² and Maria Majdan ${ }^{1}$
}

\begin{abstract}
Background: The study presents a prospective follow-up assessment of cardiovascular (CV) risk parameters in patients with rheumatoid arthritis (RA) in comparison with control subjects.

Methods: The study group consisted of 41 RA patients. The following parameters were assessed at subsequent visits [initial (T0), follow-up after 6 years (T6)]: traditional CV risk factors, carotid intima media thickness (cIMT), QTC duration, serum concentration of amino-terminal pro-brain natriuretic peptide (NT-proBNP). A comparative cIMT assessment was performed on 23 healthy controls of comparable age.

Results: The mean (SD) cIMT value in RA patients was significantly higher at T6 than at T0 [0.87 (0.21) vs 0.76 (0.15) $\mathrm{mm}, p<0.001]$, the increase in patients with atherosclerotic plaques was noted. Patients with plaques were significantly older, had higher inflammatory parameters. The mean cIMT was significantly higher in RA patients than in controls at both T6, T0 visits. Certain traditional CV risk factors exacerbated during follow up. Unfavorable metabolic parameters and significantly higher cIMT were found in male patients than in female patients at T6. During follow-up, no significant differences in NT-proBNP, QTc were found. There were no significant relationships between cIMT, NT-proBNP, QTc and parameters of disease activity at T6.

Conclusions: During the 6-year course of established RA, significant exacerbation of atherosclerosis was found, revealed by higher CIMT. A careful monitoring should be applied to patients with atherosclerotic plaques and of male gender due to higher burden of CV risk. In long-standing disease, traditional CV risk factors seem to play a key role, beyond the inflammatory activity.
\end{abstract}

Keywords: Rheumatoid arthritis, Carotid intima media thickness, Cardiovascular disease, Inflammation, Atherosclerosis

\section{Background}

Rheumatoid arthritis (RA) is a chronic, progressive, inflammatory joint disease, associated with increased risk of premature atherosclerosis and cardiovascular disease (CVD), to the similar extent as type 2 diabetes $[1,2]$. Cardiovascular (CV) death is the leading cause of mortality of patients with RA, responsible for approximately $50 \%$ of deaths [3-5]. Traditional risk factors do not fully explain the increased CV risk. Chronic inflammation and high disease activity are reportedly associated with

\footnotetext{
*Correspondence: bozena.stepniak@umlub.pl

'Department of Rheumatology and Connective Tissue Diseases, Medical

University of Lublin, ul. Jaczewskiego 8, 20-950 Lublin, Poland

Full list of author information is available at the end of the article
}

atherosclerotic burden, higher incidence of CVD, chronic heart failure (CHF), and mortality of patients with RA [6, 7].

The increased carotid intima-media thickness (cIMT) and presence of plaques are accepted as strong predictors of generalized atherosclerosis and major $\mathrm{CV}$ events in both non-RA and RA subjects $[8,9]$. Higher cIMT was reported in RA patients compared with controls [811]. Significantly higher N-terminal pro-brain natriuretic peptide (NT-pro-BNP) levels were reported in RA patients, associated with RA duration, disease activity, and inflammatory markers, suggesting a link between inflammation and cardiac stress [11-14]. A relationship was observed between NT-proBNP and cIMT [13]. The

(C) The Author(s). 2018 Open Access This article is distributed under the terms of the Creative Commons Attribution 4.0 International License (http://creativecommons.org/licenses/by/4.0/), which permits unrestricted use, distribution, and 
increased QTc values were found in RA patients, associated with parameters of disease activity, severity and inflammation $[4,15]$.

The aim of the study was a prospective assessment of $\mathrm{CV}$ risk parameters in patients with established RA, in relation to disease activity and traditional $\mathrm{CV}$ risk factors.

\section{Methods}

\section{Patients and controls}

The study group consisted of RA patients treated at the Department of Rheumatology and Connective Tissue Diseases, Medical University of Lublin. All patients met the American College of Rheumatology (ACR)/European League Against Rheumatism (EULAR) classification criteria for RA [16]. The study was conducted in full compliance with the Helsinki Declaration. The protocol was approved by the Ethics Committee of Medical University of Lublin, with the approval number KE-0254/134/2013. The informed consent for participation in the study was obtained from all participants (patients and controls), after an adequate explanation of the study design.

The study was a part of a research program involving RA patients, to prospectively analyze $\mathrm{CV}$ risk factors, both traditional and non-traditional. The assessment presented in the study was performed twice at an average interval of 6 years [73.4 (6.4) months (59-87)], at baseline visit (T0) and current follow-up after 6 years (T6).

\section{RA-related data collection}

Demographic and clinical data were obtained through structured interview, review of medical records, selfreport questionnaires, and physical examination.

Disease activity was measured using the Disease Activity Score based on evaluation of 28 joints (DAS28), calculated with the number of tender, swollen joints, erythrocyte sedimentation rate (ESR), patient's global disease activity assessment in visual analogue scale (VAS) [17].

The inflammatory burden within the 6-year course of RA was assessed as average of several results of C-reactive protein (CRP). Samples for CRP assessment were taken at consecutive visits, approximately every 6 months.

Erosive form of RA was diagnosed in patients with bone erosions in radiograms of hands and/or feet. Ability to perform daily activities was measured using modified Health Assessment Questionnaire (M-HAQ) [18].

\section{Laboratory tests}

Blood was collected after overnight fasting to determine blood cell counts, ESR, serum concentration of CRP, creatinine $(\mathrm{Cr})$, uric acid, glucose, total cholesterol (TC), high-density lipoprotein cholesterol (HDL-C), low- density lipoprotein cholesterol (LDL-C), triglycerides (TG) at the central laboratory of University Hospital. CRP was measured by immunoturbidimetric assay (upper limit $5 \mathrm{mg} / \mathrm{l}$ ). Concentrations of TC, HDL-C, TG were measured with standard enzymatic technique (BIOMAXIMA); LDL-C was calculated according to Friedewald formula. Atherogenic index (AI) calculated as ratio $\mathrm{TC} / \mathrm{HDL}-\mathrm{C}$ seems to be more appropriate to assess CV risk in RA than individual cholesterol fractions (normal AI: $<4.0$ in women and $<4.5$ in men) [19].

Serum samples were stored at $-80^{\circ} \mathrm{C}$ for further assessment of NT-pro-BNP. Measurement of NT-proBNP concentration was performed using chemiluminescent immunometric assay (IMMULITE 2000 NT-proBNP, Siemens). Reference range according to manufacturer's guidelines is up to $125 \mathrm{pg} / \mathrm{ml}$ in patients $<75$ years and up to $450 \mathrm{pg} / \mathrm{ml}$ in older; analytical sensitivity $10 \mathrm{pg} / \mathrm{ml}$. BNP and NT-proBNP levels $\geq 100 \mathrm{pg} / \mathrm{ml}$ are significantly related to cardiac morbidity and mortality [12].

\section{Metabolic and CV biomarkers}

Patients were classified as current, ex-smokers or nonsmokers. Information about concomitant diseases was taken from medical records. Physical inactivity was defined as lack of regular training. Blood pressure (BP) was assessed in a sitting position. Height and weight were measured barefoot wearing light clothes. Body mass index (BMI) was calculated as the ratio of weight and squared height.

The 10-year risk of fatal CVD using Systemic Coronary Risk Evaluation (SCORE) model was estimated in every patient, with the value $\geq 5 \%$ indicating high or very high risk. According to the EULAR recommendations, the result was multiplied by 1.5 (mSCORE) [20].

\section{Carotid intima-media thickness (cIMT) measurement}

Assessment of cIMT was performed at baseline (T0) and follow-up visit (T6) in 41 RA patients and 23 controls. All examinations were performed by the same experienced examiner, with the subject in a supine position, in a quiet, temperature-controlled room; using high-resolution B-mode ultrasound (US) (Logiq 7 GE). IMT was assessed bilaterally in three regions: common carotid artery (CCA), carotid bulb (BULB) and internal carotid artery (ICA). The analyses used the average of maximum IMT from 6 carotid segments (mean cIMT). The cIMT value $<0.6 \mathrm{~mm}$ is considered as normal, $\geq 0.9 \mathrm{~mm}$ as abnormal. The cIMT value $\geq 0.6 \mathrm{~mm}$ and $<0.9 \mathrm{~mm}$ is a marker of subclinical atherosclerosis [21]. Plaques were defined as a distinct protrusion $>1.5 \mathrm{~mm}$ into vessel lumen, with their presence as marker of advanced atherosclerosis [22]. 


\section{Electrocardiogram assessment}

The standard 12-lead transthoracic electrocardiogram (ECG) at $25 \mathrm{~mm} / \mathrm{s}$ was performed for every patient. Measurement of QTc was performed automatically with normal value $350-430 \mathrm{~ms}$ for women and $350-450 \mathrm{~ms}$ for men, respectively [23].

\section{Statistics}

Variables were tested for normality using the Kolmo gorov-Smirnov test. Group differences were tested using Student's t-test and Mann-Whitney U-test for normally and non-normally distributed parameters, respectively. Spearman's or Pearson's correlation test was used to determine association between clinical and laboratory variables. Multivariable analysis (multiple linear regression) was performed according to a forward selection procedure, introducing those variables that showed statistically significant association with certain parameters. For all tests, $P$ values $<0.05$ were considered significant.

\section{Results}

Demographic, CV risk and RA-related variables in patients A clinical characteristics of RA patients at T6 has been presented in Table 1.

The study group consisted of 41 patients with long-standing, advanced RA. The disease activity was low (DAS28 $\leq 3.2$ ) in over $50 \%$ of patients. The value of average CRP (6 years) was slightly above normal range. Most patients had an erosive form of RA and were seropositive (RF-IgM and/or anti-CCP2). Extra-articular manifestations (rheumatoid nodules, sicca syndrome, interstitial lung disease, vasculitis) in the course of the disease were observed in almost $70 \%$ of patients (Table 1).

Traditional CV risk factors occurred frequently in RA patients (Table 1).

At T6, disease-modifying antirheumatic drugs (DMARDs) were not used in one patient. Conventional synthetic DMARDs (csDMARDs) used in 39 patients included: methotrexate (MTX) in 33 patients (80.5\%), leflunomide 2 (4.9\%), antimalarials (hydroxychloroquine or chloroquine) 12 (29.3\%), cyclosporine 1 (2.4\%). Biological DMARDs (bDMARDs) included: anti-TNF in 12 (29.3\%), rituximab 14 (34.1\%) and tocilizumab $6(14.6 \%)$.

\section{Characteristics of the healthy volunteers group}

The control group consisted of 23 healthy volunteers: 15 women $(65.2 \%)$ and 8 men (34.8\%), with the mean (SD) age of 49.6 (6.2) years (39-62), BMI 25.3 (3.1) $\mathrm{kg} / \mathrm{m}^{2}$ (22.2-31.2). At T6, 10 (43.5\%) controls were current/ex-smoker and $6(26.1 \%)$ controls had arterial hypertension.
Table 1 Clinical characteristics of 41 RA patients at follow-up visit (T6)

\begin{tabular}{ll}
\hline Variables & Results \\
\hline Demographic variables: & \\
Age, years & $53.3(8.7)(28-68)$ \\
Gender, F/M & $34(82.9) / 7(17)$ \\
Cardiovascular risk factors: & \\
Family history of CVD & $20(48.8)$ \\
Current/Ex-smokers & $23(56.1)$ \\
Hypertension & $17(41.5)$ \\
Diabetes & $3(7.3)$ \\
CKD3a (eGFR 45-59 ml/min/1.73 m²) & $2(4.9)$ \\
Al abnormal & $11(26.8)$ \\
BMI > 30 kg/m ${ }^{2}$ & $5(12.2)$ \\
Physical inactivity & $23(56.1)$ \\
RA related variables & \\
Disease duration, years & $19.2(9.2)(8-45)$ \\
Erosions (X-ray of hands/feet) & $35(85.4)$ \\
Extra-articular symptoms & $28(68.3)$ \\
Positive RF-lgM & $33(80.5)$ \\
Positive anti-CCP2 & $30(73.2)$ \\
ESR, mm/h & $19.2(16.0)(2-64)$ \\
CRP, mg/l & $14.35(37.5)(0.1-228.5)$ \\
Average CRP (6 years), mg/l & $11.02(9.9)(0.9-37.3)$ \\
Low RA activity (DAS28 3.2$)$ & $23(56.1)$ \\
Current glucocorticoid use & $18(43.9)$ \\
Current conventional synthetic DMARD & $39(95.1)$ \\
Current biological DMARD & $32(78.1)$ \\
\hline & \\
Dat are presented as mean (SD) (rnge) or number & \\
\hline &
\end{tabular}

Data are presented as mean (SD) (range) or number (\%); $A$ l atherogenic index Anti-CCP2 anti-cyclic citrullinated peptide antibodies, BMI body mass index, CVD cardiovascular disease, CKD chronic kidney disease, CRP C-reactive protein; DAS28 disease activity score in 28 joints, DMARD disease modifying antirheumatic drug, eGFR estimated glomerular filtration rate, ESR erythrocyte sedimentation rate, $R F-\lg M$ IgM rheumatoid factor

The mean age and BMI did not differ statistically between patients and controls.

\section{Comparison of cIMT between RA patients and controls}

The mean value of cIMT was significantly higher in patients than in controls at both visits: current (T6) and baseline (T0) (Table 2).

The significant increase of cIMT value during the 6-year follow-up was noted in both RA patients $(p<0.001)$ and controls $(p<0.001)$. The average increase of cIMT between T0 and T6 (delta IMT) was not significantly different between patients and controls $[0.11(0.18)$ vs 0.17 (0.09) $\mathrm{mm}, \mathrm{NS}$ ]. Carotid plaques were observed more often in patients than in controls at both T0 and T6 (statistically nonsignificant) (Table 2). 
Table 2 Comparison of CIMT and atherosclerotic plaques at T0 and T6 visits in the group of RA patients and controls

\begin{tabular}{|c|c|c|c|c|c|}
\hline Parameters & Group & T0 & $p$ & T6 & $p$ \\
\hline \multirow[t]{2}{*}{ Mean cIMT, mm } & RA patients & $0.76(0.15)(0.43-1.2)$ & $<0.001$ & $0.87(0.21)(0.57-1.77)$ & 0.03 \\
\hline & Controls & 0.59 (0.11) (0.4-0.87) & & $0.76(0.11)(0.61-1.07)$ & \\
\hline \multirow[t]{2}{*}{ Abnormal cIMT ( $\geq 0.9$ mm) } & RA patients & $9(21.9)$ & 0.6 & $14(34.1)$ & 0.6 \\
\hline & Controls & $1(4.3)$ & & $3(13.0)$ & \\
\hline \multirow[t]{2}{*}{ Atherosclerotic plaques presence } & RA patients & $6(14.6)$ & NS & $10(24.4)$ & NS \\
\hline & Controls & $1(4.3)$ & & $4(17.4)$ & \\
\hline \multirow[t]{2}{*}{ Bilateral atherosclerotic plaques presence } & RA patients & $3(3.7)$ & NS & $7(17.1)$ & NS \\
\hline & Controls & 0 & & $1(4.3)$ & \\
\hline
\end{tabular}

Data are presented as mean (SD) (range), cIMT carotid intima media thickness

\section{Comparison of clinical and laboratory parameters at T0 and T6 in RA patients}

Disease activity assessed with DAS28 diminished significantly between T0 and T6 (Table 3).

Significantly higher cIMT value was found in RA patients at T6 compared with T0 [0.87 (0.21) mm vs 0.76 (0.15), $p<0.001$ ] (Table 3).

Most patients had increased cIMT value at both T6 and T0 assessments (Fig. 1). However, during the 6-year follow-up, an increase in the number of patients with defined atherosclerosis (cIMT $\geq 0,9 \mathrm{~mm}$ )
(Fig. 1) and atherosclerotic plaques (Table 3) was observed (statistically nonsignificant). The number of patients with bilateral plaques increased between T0 and T6 (statistically nonsignificant) (Fig. 2).

Serum NT-proBNP concentration and QTc duration did not change significantly between T0 and T6 (Table 3).

Certain traditional CV risk factors (serum uric acid, BMI) and mSCORE exacerbated between T0 and T6 (Table 3). The number of patients with high/ very high risk of $\mathrm{CV}$ death increased significantly (Table 3).

Table 3 Comparison of clinical and laboratory parameters at T0 and T6 in 41 RA patients

\begin{tabular}{|c|c|c|c|}
\hline Variables & T0 & T6 & $p$ \\
\hline \multicolumn{4}{|l|}{ Clinical parameters } \\
\hline DAS28 & 4.46 (1.15) (2.58-6.55) & 3.33 (1.62) (0.66-6.88) & $<0.001$ \\
\hline $\mathrm{M}-\mathrm{HAQ}$ & $1.22(0.52)(0-2.38)$ & $1.31(0.52)(0.25-2.38)$ & NS \\
\hline $\mathrm{SBP}, \mathrm{mmHg}$ & 123.8 (13.5) (90-160) & $128.2(13.6)(105-160)$ & NS \\
\hline $\mathrm{BMI}, \mathrm{kg} / \mathrm{m}^{2}$ & 24.8 (3.1) (18.6-29.95) & 25.9 (3.4) (17.3-33.2) & 0.002 \\
\hline $\mathrm{BMI}>30 \mathrm{~kg} / \mathrm{m}^{2}$ & 0 & $5(12.2)$ & 0.03 \\
\hline mSCORE, \% & $0.98(2.1)(0-12)$ & 2.99 (3.9) (0-18) & $<0.001$ \\
\hline High/very high CVD risk & $2(4.9)$ & $10(24.4)$ & 0.01 \\
\hline QTc, ms & 341.9 (60.8) (187-446) & $358.8(59.5)(175-453)$ & NS \\
\hline cIMT, mm & $0.76(0.15)(0.43-1.2)$ & 0.87 (0.21) (0.57-1.77) & $<0.001$ \\
\hline Carotid plaques presence & $6(14.6)$ & $10(24.4)$ & NS \\
\hline \multicolumn{4}{|l|}{ Laboratory parameters } \\
\hline NT-proBNP, pg/ml & 88.9 (78) (12.2-351.2) & 126.6 (186.5) (20.1-1175) & NS \\
\hline Glucose, mg/dl & 89.3 (12.9) (54-120) & 88.8 (14.5) (67-146) & NS \\
\hline $\mathrm{TC}, \mathrm{mg} / \mathrm{dl}$ & 200.6 (42) (135-325) & $206.3(7.2)(101-318)$ & NS \\
\hline $\mathrm{HDL}-\mathrm{C}, \mathrm{mg} / \mathrm{dl}$ & 59.6 (14.2) (39-87) & $60.3(16.2)(30-102)$ & NS \\
\hline $\mathrm{LDL}-\mathrm{C}, \mathrm{mg} / \mathrm{dl}$ & 119.7 (36.2) (47-232) & 120.8 (34.4) (41-190) & NS \\
\hline Triglycerides, mg/dl & 106.5 (45.6) (43-214) & 113.0 (53.5) (23-251) & NS \\
\hline $\mathrm{Al}(\mathrm{TC} / \mathrm{HDL}-\mathrm{C})$ & $3.48(0.81)(2.1-5.5)$ & $3.53(0.84)(2.0-5.8)$ & NS \\
\hline Serum creatinine, mg/dl & $0.68(0.15)(0.4-1.0)$ & $0.66(0.15)(0.4-1.0)$ & NS \\
\hline Serum uric acid, mg/dl & $4.0(0.9)(2.2-5.8)$ & $4.5(1.1)(2.5-7)$ & $<0.05$ \\
\hline
\end{tabular}




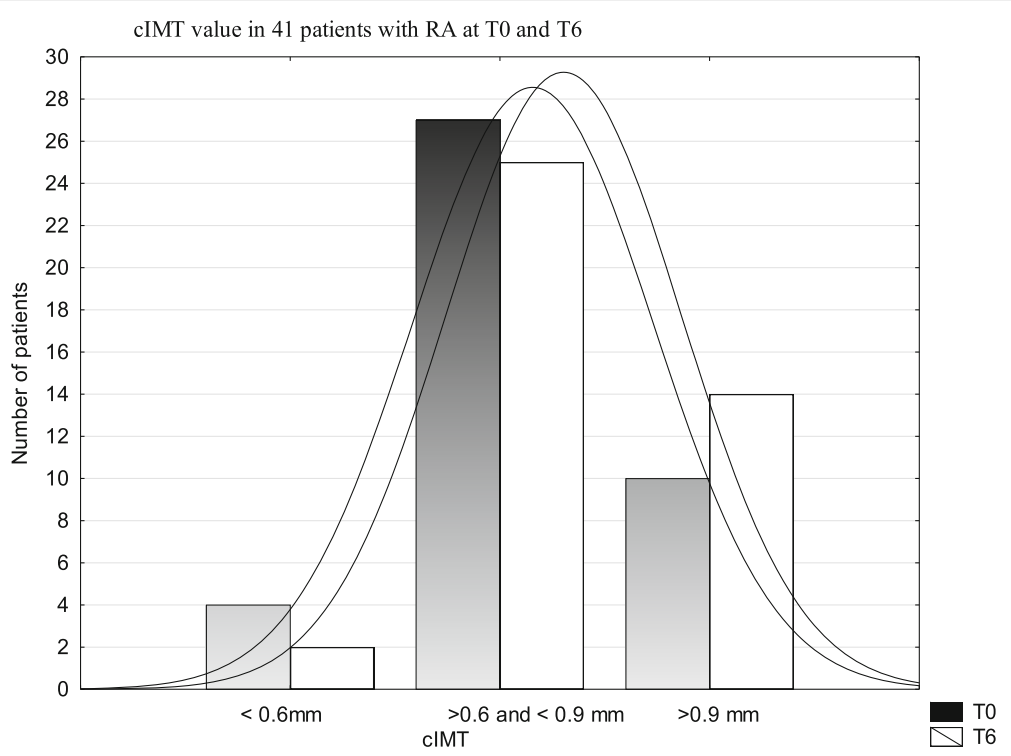

Fig. 1 Comparison of CIMT value in 41 patients with RA at T0 and T6

The other clinical and laboratory parameters did not change significantly (Table 3 ).

\section{Relationship between CV risk parameters at T0 and T6 in RA patients}

There was a strong relationship between the mean cIMT values assessed in RA patients at T6 and T0 $(p<0.001)$. In multiple linear regression analysis, significant association at T0 was confirmed between cIMT and SCORE ( $p=$ $0.001)$, NT-proBNP $(p=0.04)$. This relationship was not observed at T6. There were no significant relationships between cIMT, NT-proBNP, OTc and parameters of current disease activity or inflammation as well as average CRP.

\section{Comparison of patients with and without atherosclerotic plaques}

Patients with atherosclerotic plaques at T6 were older [59.0 (5.7) vs 51.5 (8.7), $p=0.02$ ], had significantly higher CRP concentration [40.6 (72) vs 6.2 (9.5), $p=0.01$ ] and white blood cell count (WBC) [7.6 (2.6) vs 5.5 (1.6), $p=$ 0.006].

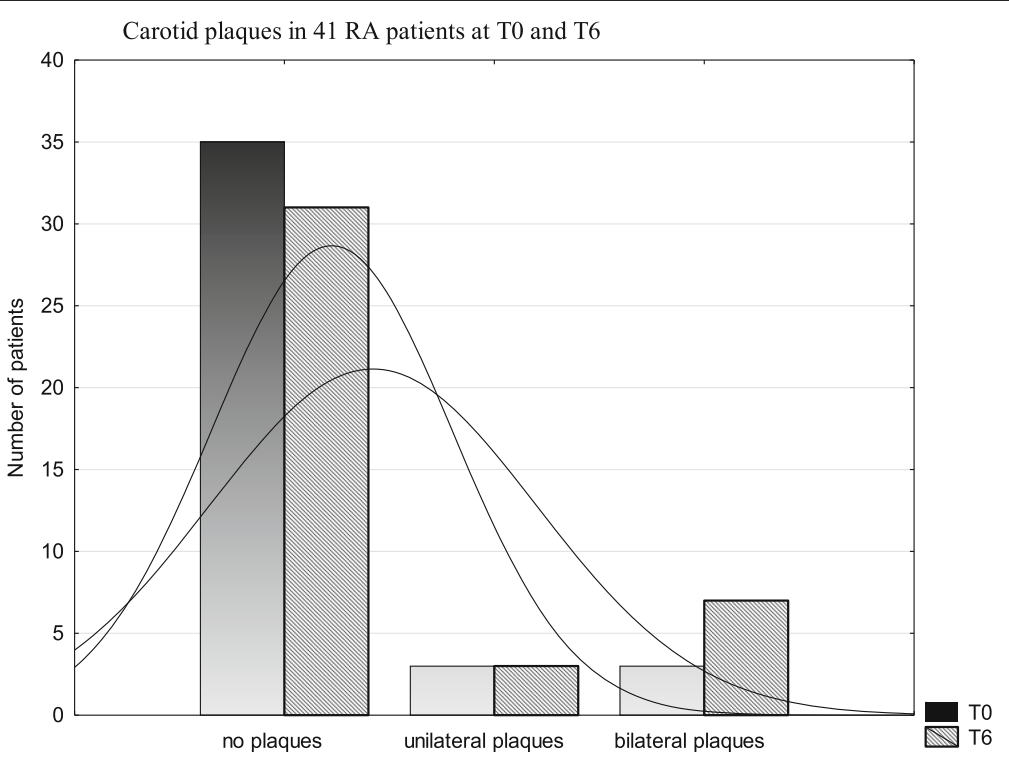

Fig. 2 Comparison of atherosclerotic carotid plaques in 41 RA patients at T0 and T6 


\section{Comparison of male and female RA patient}

At T0, the mean cIMT value was non-significantly higher in men than in women with RA $[0.82(0.2)$ vs $0.75(0.14), \mathrm{NS}]$. However, at T6, unfavorable CV and metabolic parameters were found in male compared with female patients: significantly higher cIMT [1.1 (0.33) vs $0.82(0.14), p<0.001]$, SCORE [6.0 (6.1) vs 2.3 (2.9), $p=0.04$ ], uric acid [5.5 (1.0) vs 4.3 (1.0), $p=0.007$ ] and lower HDL-C [44.3 (10.7) vs 63.1 (15.4), $p=0.007]$.

In control subjects, the mean cIMT at T6 did not differ significantly between men and women $[0.77(0.13)$ vs 0.76 (0.1), NS].

\section{Discussion}

In this prospective, 6-year follow-up study, exacerbation of atherosclerosis was observed in patients with advanced RA, revealed by a significant increase of cIMT value and an increase in patients with atherosclerotic plaques (non-significant). Patients with atherosclerotic plaques showed significantly higher inflammatory parameters than patients without plaques, suggesting association between inflammatory burden and plaques presence. Especially the presence of bilateral plaques is noteworthy due to significantly higher $\mathrm{CV}$ risk and the quadrupled risk of acute myocardial syndrome [24].

Exacerbation of atherosclerosis occurred despite effective control of RA activity. Disease activity according to DAS28 decreased significantly during follow-up, which was associated with active DMARDs treatment. Simultaneously deterioration was found in certain traditional $\mathrm{CV}$ risk factors. During follow-up the risk of $\mathrm{CV}$ death increased significantly and 25\% of patients had high/very high risk of fatal CVD.

The current cIMT was significantly associated only with baseline cIMT assessed 6 years before. No significant relationship was found between current cIMT, NT-proBNP, QTc values and disease activity parameters. The results suggest, that initial state of CV system had a profound effect on further CV risk in the course of RA.

Exacerbation of atherosclerosis, revealed by higher cIMT, was also found in the control group. The cIMT increases were comparable in both groups (patients and controls), which suggests an effect of physiological aging. Similar results were presented in a follow-up study, in which, after 5-year observation, significantly higher cIMT was found in both early RA patients and controls, atherosclerotic plaques were not mentioned [11].

Atherosclerotic plaques were shown to be more predictive of $\mathrm{CV}$ events than elevated cIMT, since cIMT value may be associated with arterial wall aging, body size, muscularity [25]. New or progressive carotid plaques were strongly associated with markers of inflammation and disease activity (CRP, swollen joints count) [25]. It seems that in RA, an additional, maladaptive arterial wall remodeling increases risk of plaque rapture which could explain highly increased CV risk despite a seemingly normal cIMT [26]. According to Semb et al., patients with RA had numerically more atherosclerotic plaques than controls, associated with the presence of RA, but not with the level of RA activity. However, patients in remission and controls had more stable plaques than patients with active disease, pointing to importance of achieving remission [27].

Several hypotheses have been proposed to explain the accelerated atherosclerosis in RA. Thus far, there is no evidence that RA-associated single-nucleotide polymorphisms (SNP) as a group are associated with coronary artery disease [28].

The greater burden of traditional CV risk factors is suggested among RA patients [28, 29]. It seems that classical CV risk factors may be important for generation and progression of stable atherosclerosis, whereas local and systemic high-grade inflammation may contribute to plaque instability and higher rates of acute $\mathrm{CV}$ syndromes [30]. Dalbeni et al. found that only age was consistently associated with cIMT and atherosclerotic plaques as major determinant of subclinical atherosclerosis [31]. Male gender is reported to have an impact on cIMT in addition to disease activity parameters [8]. According to data in literature, on average cIMT is higher in men than in women and increases with age [32].

In this study, cIMT value, which was comparable in male and female patients at initial (T0) assessment, became significantly higher in males at the 6-year follow-up. Simultaneously, a higher burden of traditional $\mathrm{CV}$ factors and risk of $\mathrm{CV}$ death was found in men with RA.

Systemic, immune-mediated inflammation significantly contributes to accelerated atherosclerosis [6]. Effective control of inflammation as a result of DMARDs treatment is associated with CV risk reduction. Significantly lower cIMT was reported in RA patients treated with MTX and correlated with MTX dosage [33]. Significant cIMT reduction was reported in RA patients treated with TNF inhibitors and steadily responsive to therapy [34].

In established RA, NT-proBNP was associated with the disease activity, duration, as well as cIMT $[2,5,12$, 13]. It was suggested that increased NT-proBNP could reflect silent myocardial stress associated with transient ischemia due to atherosclerosis [13]. It was reported that in RA patients without evident $\mathrm{CHF}$, treatment with TNF inhibitor decreased NT-proBNP, suggesting the link between inflammation and cardiac stress [35]. In this study, non-significant increase of NT-proBNP was observed during follow-up, with no clinical symptoms of CHF. The significant association between NT-proBNP and cIMT at T0 was not found at follow-up, which may be considered as a result of active DMARDs treatment. 
It seems that traditional $\mathrm{CV}$ risk factors in association with high-grade inflammation are responsible for acceleration of atherosclerosis in early course of RA. The results of our study indicate that at follow-up the burden of atherosclerosis is still higher in RA patients than in healthy controls and constantly related to the disease activity. Traditional CV risk factors seem to be important in long-standing advanced RA.

The careful diagnosis and management of $\mathrm{CV}$ risk factors should be considered for all RA patients, especially those with atherosclerotic plaques should be treated as high-risk patients.

The main strength of our present study is its prospective design. The same two groups of patients and controls were assessed with a 6-year interval. The detailed characteristics of patients considered all aspects of RA pathology. Another strength is the same specialist who performed all US measurements, thereby eliminating any interpersonal variations.

There are some limitations of the study, including quite small groups of patients and controls. The score used in the study (DAS28) presents only the current disease activity and does not reflect the activity burden over the 6 years. Therefore, further evaluation should be performed on a larger group of patients, considering assessment of the activity burden with other methods.

\section{Conclusions}

In this prospective, 6-year follow-up study of patients with established RA, significant exacerbation of atherosclerosis was found, as revealed by higher cIMT and increase in patients with atherosclerotic plaques, despite significant reduction of disease activity. Unfavorable metabolic parameters and significantly higher cIMT in male compared with female patients were noted. The mean cIMT was significantly higher in RA patients than in controls at both assessments. Tight control of inflammation could be an effective method to reduce $\mathrm{CV}$ risk. However, in long-standing disease traditional CV risk factors seem to play a key role, beyond inflammatory activity of the disease.

\section{Abbreviations}

ACR: American College of Rheumatology; Al: Atherogenic index; bDMARD: biological DMARD; BMI: Body mass index; BP: Blood pressure; BULB: Carotid bulb; CCA: Common carotid artery; CHF: Congestive heart failure; CIMT: carotid intima-media thickness; Cr: Creatinine; CRP: C-reactive protein; CsDMARD: conventional synthetic DMARD; CV: Cardiovascular; CVD: Cardiovascular disease; DAS28: Disease Activity Score evaluated in 28 joints; DMARD: Disease-modifying antirheumatic drug;

ECG: Electrocardiogram; ESR: Erythrocyte sedimentation rate;

EULAR: European League Against Rheumatism; HDL-C: High-density lipoprotein cholesterol; ICA: Internal carotid artery; LDL-C: Low-density lipoprotein cholesterol; M-HAQ: Modified Health Assessment Questionnaire; NT-proBNP: N-terminal pro-brain natriuretic peptide; QTc: Heart ratecorrected QT interval; RA: Rheumatoid arthritis; SCORE: Systemic Coronary Risk Evaluation; TC: Total cholesterol; TG: Triglycerides; TNF: Tumor necrosis factor; US: Ultrasound; VAS: Visual analogue scale
Funding

Medical University of Lublin Grant.

\section{Availability of data and materials}

The datasets used and/or analyzed during the current study are available from the corresponding author on reasonable request.

\section{Authors' contributions}

BTS contributed to conception and design, contributed to acquisition, analysis and interpretation of data, was a major contributor in writing the manuscript. MP contributed to acquisition, analysis, performed the statistical analyses. RZ contributed to acquisition, analysis and interpretation of data. ADZ performed all the ultrasound examinations, contributed to analysis and interpretation of data. MM contributed to conception and design, contributed to interpretation of data. All authors read and approved the final manuscript.

\section{Ethics approval and consent to participate}

The study has been approved by the appropriate ethics committees and has, therefore, been performed in accordance with the ethical standards laid down in the 1964 Declaration of Helsinki and its later amendments. All persons gave their informed consent prior to their inclusion in the study.

\section{Consent for publication}

Not applicable.

\section{Competing interests}

The authors declare that they have no competing interests.

\section{Publisher's Note}

Springer Nature remains neutral with regard to jurisdictional claims in published maps and institutional affiliations.

\section{Author details}

${ }^{1}$ Department of Rheumatology and Connective Tissue Diseases, Medical University of Lublin, ul. Jaczewskiego 8, 20-950 Lublin, Poland. Department of Interventional Radiology and Neuroradiology, Medical University of Lublin, ul. Jaczewskiego 8, 20-950 Lublin, Poland.

Received: 8 January 2018 Accepted: 24 July 2018

Published: 1 August 2018

\section{References}

1. Corrales A, Dessein PH, Tsang L, Pina T, Blanco R, Gonzalez-Juanatey C, et al. Carotid artery plaque in women with rheumatoid arthritis and low estimated cardiovascular disease risk: a cross-sectional study. Arthritis Res Ther. 2015;17:55

2. Provan SA, Angel K, Odegård S, Mowinckel P, Atar D, Kvien TK. The association between disease activity and NT-proBNP in 238 patients with rheumatoid arthritis: a 10-year longitudinal study. Arthritis Res Ther. 2008;10:R70.

3. Pope JE, Nevskaya T, Barra L, Parraga G. Carotid artery atherosclerosis in patients with active rheumatoid arthritis: predictors of plaque occurrence and progression over 24 weeks. Open Rheumatol J. 2016;10:49-59.

4. Lazzerini PE, Capecchi PL, Acampa M, Galeazzi M, Laghi-Pasini F. Arrhythmic risk in rheumatoid arthritis: the driving role of systemic inflammation. Autoimmun Rev. 2014:13:936-44.

5. Provan S, Angel K, Semb AG, Atar D, Kvien TK. NT-proBNP predicts mortality in patients with rheumatoid arthritis: results from 10-year follow-up of the EURIDISS study. Ann Rheum Dis. 2010;69:1946-50.

6. Zhang J, Chen L, Delzell E, Muntner P, Hillegass WB, Safford MM, et al. The association between inflammatory markers, serum lipids and the risk of cardiovascular events in patients with rheumatoid arthritis. Ann Rheum Dis. 2014;73:1301-8.

7. Navarro-Millán I, Yang S, DuVall SL, Chen L, Baddley J, Cannon GW, et al. Association of hyperlipidaemia, inflammation and serological status and coronary heart disease among patients with rheumatoid arthritis: data from the National Veterans Health Administration. Ann Rheum Dis. 2016;75:341-7. 
8. Ambrosino P, Lupoli R, Di Minno A, Tasso M, Peluso R, Di Minno MN. Subclinical atherosclerosis in patients with rheumatoid arthritis. A metaanalysis of literature studies. Thromb Haemost. 2015;113:916-30.

9. Wang P, Guan SY, Xu SZ, Li HM, Leng RX, Li XP, et al. Increased carotid intima-media thickness in rheumatoid arthritis: an update meta-analysis. Clin Rheumatol. 2016:35:315-23.

10. Targońska-Stępniak B, Drelich-Zbroja A, Majdan M. The relationship between carotid intima-media thickness and the activity of rheumatoid arthritis. J Clin Rheumatol. 2011;17:249-55.

11. Södergren A, Karp K, Bengtsson C, Möller B, Rantapää-Dahlqvist S, WållbergJonsson S. The extent of subclinical atherosclerosis is partially predicted by the inflammatory load: a prospective study over 5 years in patients with rheumatoid arthritis and matched controls. J Rheumatol. 2015;42:935-42.

12. Mirjafari $H$, Welsh $P$, Verstappen $S M$, Wilson $P$, Marshall $T$, Edlin $H$, et al. $\mathrm{N}$-terminal pro-brain-type natriuretic peptide (NT-pro-BNP) and mortality risk in early inflammatory polyarthritis: results from the Norfolk arthritis registry (NOAR). Ann Rheum Dis. 2014;73:684-90.

13. Targońska-Stępniak B, Majdan M. Amino-terminal pro-brain natriuretic peptide as a prognostic marker in patients with rheumatoid arthritis. Clin Rheumatol. 2011;30:61-9.

14. Avouac J, Meune C, Chenevier-Gobeaux C, Dieudé P, Borderie D, Lefevre $\mathrm{G}$, et al. Inflammation and disease activity are associated with high circulating cardiac markers in rheumatoid arthritis independently of traditional cardiovascular risk factors. J Rheumatol. 2014;41:248-55.

15. Lazzerini PE, Capecchi PL, Laghi-Pasini F. Systemic inflammation and arrhythmic risk: lessons from rheumatoid arthritis. Eur Heart J. 2017;38:1717-27.

16. Aletaha D, Neogi T, Silman AJ, Funovits J, Felson DT, Bingham CO 3rd, et al. 2010 Rheumatoid arthritis classification criteria: an American College of Rheumatology/European League Against Rheumatism collaborative initiative. Arthritis Rheum. 2010;62:2569-81.

17. Prevoo ML, van't Hof MA, Kuper HH, van Leeuwen AN, van de Putte LB, van Riel PL. Modified disease activity scores that include twenty-eight-joint counts: development and validation in a prospective longitudinal study of patients with rheumatoid arthritis. Arthritis Rheum. 1995:38:44-8.

18. Pincus T, Sokka T, Kautiainen H. Further development of a physical function scale on a MDHAQ [corrected] for standard care of patients with rheumatic diseases. J Rheumatol. 2005;32:1432-9.

19. Popa CD, Arts E, Fransen J, van Riel PLCM. Atherogenic Index and highdensity lipoprotein cholesterol as cardiovascular risk determinants in Rheumatoid Arthritis: The Impact of Therapy with Biologicals. Mediators Inflamm. 2012;2012:785946.

20. Peters MJL, Symmons DPM, McCarey D, Dijkmans BAC, Nicola P, Kvien TK, et al. EULAR evidence-based recommendations for cardiovascular risk management in patients with rheumatoid arthritis and other forms of inflammatory arthritis. Ann Rheum Dis. 2010;69:325-31.

21. Gonzalez-Gay MA, Gonzalez-Juanatey C, Vazquez-Rodriguez TR, Martin J, Llorca J. Endothelial dysfunction, carotid intima-media thickness and accelerated atherosclerosis in rheumatoid arthritis. Semin Arthritis Rheum. 2008;38:67-70

22. Gonzalez-Juanatey C, Llorca J, Martin J, Gonzalez-Gay MA. Carotid intima-media thickness predicts the development of cardiovascular events in patients with rheumatoid arthritis. Semin Arthritis Rheum. 2009:38:366-71.

23. Goldenberg I, Moss AJ, Zareba W. QT interval: how to measure it and what is "normal". J Cardiovasc Electrophysiol. 2006:17:333-6.

24. Van Sijl AM, Van Den Hurk K, Peters MJ, Van Halm VP, Nijpels G, Stehouwer CDA, et al. Different type of carotid Arterial Wall remodeling in rheumatoid arthritis compared with healthy subjects: a case-control study. J Rheumatol. 2012;39:2261-6.

25. Giles JT, Post WS, Blumenthal RS, Polak J, Petri M, Gelber AC, et al. Longitudinal predictors of progression of carotid atherosclerosis in rheumatoid arthritis. Arthritis Rheum. 2011;63:3216-25.

26. Evans MR, Escalante A, Battafarano DF, Freeman GL, O'Leary DH, del Rincón I. Carotid atherosclerosis predicts incident acute coronary syndromes in rheumatoid arthritis. Arthritis Rheum. 2011;63:1211-20.

27. Semb AG, Rollefstad S, Provan SA, Kvien TK, Stranden E, Olsen IC, et al. Carotid plaque characteristics and disease activity in rheumatoid arthritis. J Rheumatol. 2013;40:359-68.

28. Jansen H, Willenborg C, Lieb W, Zeng L, Ferrario PG, Loley C, et al. Rheumatoid arthritis and coronary artery disease: genetic analyses do not support a causal relation. J Rheumatol. 2017;44:4-10.
29. Baghdadi LR, Woodman RJ, Shanahan EM, Mangoni AA. The impact of traditional cardiovascular risk factors on cardiovascular outcomes in patients with rheumatoid arthritis: a systematic review and meta-analysis. PLoS One. 2015:10:e0117952.

30. Kitas GD, Gabriel SE. Cardiovascular disease in rheumatoid arthritis: state of the art and future perspectives. Ann Rheum Dis. 2011;70:8-14.

31. Dalbeni A, Giollo A, Tagetti A, Atanasio S, Orsolini G, Cioffi G, et al. Traditional cardiovascular risk factors or inflammation: which factors accelerate atherosclerosis in arthritis patients? Int J Cardiol. 2017;236:488-92.

32. Roman MJ, Naqvi TZ, Gardin JM, et al. American society of echocardiography report. Clinical application of noninvasive vascular ultrasound in cardiovascular risk stratification: a report from the American Society of Echocardiography and the society for vascular medicine and biology. Vasc Med. 2006;11:201-11.

33. Kim HJ, Kim MJ, Lee CK, Hong YH. Effects of methotrexate on carotid intima-media thickness in patients with rheumatoid arthritis. J Korean Med Sci. 2015;30:1589-96.

34. Del Porto F, Laganà B, Lai S, Nofroni I, Tinti F, Vitale M, et al. Response to anti-tumour necrosis factor alpha blockade is associated with reduction of carotid intima-media thickness in patients with active rheumatoid arthritis. Rheumatology (Oxford). 2007;46:1111-5.

35. Peters MJ, Welsh P, Mclnnes IB, Wolbink G, Dijkmans BA, Sattar N, et al. Tumour necrosis factor $\{$ alpha\} blockade reduces circulating $\mathrm{N}$-terminal probrain natriuretic peptide levels in patients with active rheumatoid arthritis: results from a prospective cohort study. Ann Rheum Dis. 2010;69:1281-5.
Ready to submit your research? Choose BMC and benefit from:

- fast, convenient online submission

- thorough peer review by experienced researchers in your field

- rapid publication on acceptance

- support for research data, including large and complex data types

- gold Open Access which fosters wider collaboration and increased citations

- maximum visibility for your research: over $100 \mathrm{M}$ website views per year

At $\mathrm{BMC}$, research is always in progress.

Learn more biomedcentral.com/submissions 